\title{
Comparative genomics of Streptococcus parauberis: new target for molecular identification of serotype III
}

\author{
Yolanda Torres-Corral $^{1}$ (D) $\cdot$ Ysabel Santos $^{1}$ (D) \\ Received: 19 February 2020 / Revised: 2 May 2020 / Accepted: 12 May 2020 / Published online: 21 May 2020 \\ (C) Springer-Verlag GmbH Germany, part of Springer Nature 2020
}

\begin{abstract}
This paper describes the predicted structure for the cps loci involved in capsule biosynthesis for Streptococcus parauberis serotypes III, IV, and V. Based on the specific serotype regions I, II, and III, a multiplex PCR protocol (mPCR) was designed to differentiate the main serotypes causing fish diseases. A real-time PCR method (qPCR) is also described to identify S. parauberis of serotype III in bacterial cultures and fish tissues. In silico and in vitro analyses revealed that both methods have a $100 \%$ specificity. The mPCR assay was optimized for the detection of $S$. parauberis strains of subtypes Ia (amplicon size $213 \mathrm{bp}$ ), subtypes Ib and Ic (both amplicon size $303 \mathrm{bp}$ ), serotype II (amplicon size $403 \mathrm{bp}$ ), and serotype III (amplicon size $130 \mathrm{bp}$ ) from bacterial cultures. The qPCR assay was optimized for the identification and quantification of S. parauberis serotype III strains in bacterial cultures and fish tissues. This assay achieved a sensitivity of $2.67 \times 10^{2}$ gene copies (equivalent to $3.8 \times$ $\left.10^{-9} \mathrm{ng} / \mu \mathrm{l}\right)$ using pure bacterial cultures of S. parauberis serotype III and $1.76 \times 10^{2}$ gene copies in fish tissues experimentally and naturally infected with S. parauberis of the serotype III. The specificity and sensitivity of the protocols described in this study suggest that these methods could be used for diagnostic and/or epidemiological purposes in clinical diagnostic laboratories.
\end{abstract}

\section{Key Points}

- Structure of loci cps for S. parauberis of serotypes III, IV and V was described.

- mPCR to differentiate $S$. parauberis serotypes causing disease in fish was optimized.

- qPCR assay to quantify strains of S. parauberis serotype III in fish tissues.

Keywords Real-time PCR · Streptococcus parauberis · Serotypes III · IV and V · Genomic analyses · Typing

\section{Introduction}

Streptococcus parauberis is a Gram-positive bacterium responsible for streptococcosis, a disease that causes major

Electronic supplementary material The online version of this article (https://doi.org/10.1007/s00253-020-10683-z) contains supplementary material, which is available to authorized users.

Yolanda Torres-Corral

yolanda.torres.corral@gmail.com

Ysabel Santos

ysabel.santos@usc.es

1 Departamento de Microbiología, Instituto de Análisis Químico y Biológico (IAQBUS), Universidad de Santiago de Compostela, 15782 Santiago de Compostela, Spain economic losses in the aquaculture sector. This pathology affects the central nervous system of fish, resulting in septicaemia and meningoencephalitis. S. parauberis was initially described as an aetiological agent of bovine mastitis (Williams and Collins 1990; Khan et al. 2003). Since then, $S$. parauberis has become an emerging pathogen in the aquaculture industry responsible for streptococcosis epizootics mainly in turbot (Scophthalmus maximus) farmed in Spain (Domenech et al. 1996), in flounder (Paralichthys olivaceus and Platichthys stellatus) farmed in Asian countries (Aoki et al. 1990; Baeck et al. 2006; Cho et al. 2008), and sea bass (Sebastes ventricosus) farmed in Japan (Oguro et al. 2014). Recently, S. parauberis was also isolated from wild striped sea bass (Morone saxatilis) in North America (USA) (Haines et al. 2013). Diversity among $S$. parauberis isolates was reported at genotypic and serological levels (Austin and Austin 2016). 
Polysaccharide capsules are structures found on the cell surface of many bacterial species. These structures play an important role in pathogenicity, immune system escape, serum resistance, inflammation, adhesion, biofilm formation, and antigenicity, which may be used for bacterial serotyping (Okura et al. 2013; Rochat et al. 2017). The diversity of capsular polysaccharide (CPS) structure of $S$. parauberis, due to a variety of sugars and glycosidic linkages, led to the description of different serotypes designated as types I, II, and III (Kanai et al. 2009; Kanai et al. 2015; Tu et al. 2015a; Torres Corral et al. 2019). Serotype I was further subdivided into three subtypes, designated Ia, Ib, and Ic (Kanai et al. 2015; $\mathrm{Tu}$ et al. 2015a). S. parauberis isolates agglutinating with antibodies of serotypes I and II were also described and designated as non-typable strains (Kanai et al. 2015; Tu et al. 2015a).

The genes responsible for the synthesis of CPS are typically clustered in a single locus (locus cps) on the chromosome (Okura et al. 2013; Tu et al. 2015a). The genetic structure of the loci cps in S. parauberis has been determined in serotypes I and II (Tu et al. 2015a). In these serotypes a cassette-like structure was observed, in which the conserved regions flanked serotype-specific genes. At the conserved $5^{\prime}$ end of the cps regions, a group of five regulatory genes (lysR, cpsA. cps $B, \operatorname{cps} C$, and $c p s D$ ) and one processing gene (cpsE) were found, while at the conserved $3^{\prime}$ end, one regulatory gene $(\operatorname{cps} Q)$ and one hypothetical gene were found. The middle region (variable region) of the locus cps comprises genes encoding enzymes such as glycosyltransferases, acetyltransferases, aminotransferases, and modifying enzymes (Tu et al. 2015a).

This serological diversity might have important consequences for the selection of suitable candidate strains for vaccine development, in monitoring studies, epidemiological surveillance, disease control, as well as for a better understanding of host virulence and resistance traits. Serotyping is, therefore, a necessary tool for the diagnosis and epidemiological surveillance of streptococcosis caused by S. parauberis. However, serotyping has important limitations such as sensitivity, specificity, and the need to obtain antisera from animals. These limitations added to the recent determination of complete genomic sequences of a wide variety of bacteria have allowed the development of new molecular methods based on polymerase chain reaction (PCR) for bacterial typing. In recent years, different PCR bacterial typing protocols have emerged as an alternative for the use of antisera in pathogenic fish species, such as Streptococcus agalactiae (Demczuk et al. 2017; Kannika et al. 2017; Shoemaker et al. 2017), Flavobacterium psychrophylum (Rochat et al. 2017), S. parauberis (Tu et al. 2015b), and Lactococcus garvieae (Ohbayashi et al. 2017).

In the present study, genomic sequences of $S$. parauberis of different serotypes, hosts, and geographic regions were compared to determine the genetic structure of the locus cps of the recently described serotype III of $S$. parauberis (Torres Corral et al. 2019). The serotype-specific region of S. parauberis serotype III was used as a target to develop real-time PCR (qPCR) protocol to identify, detect, and quantify strains of $S$. parauberis serotype III. The specificity and efficacy of this assay was evaluated in silico and in vitro using target and non-target bacterial cultures and fish tissue samples. In addition, a serotyping scheme based on multiplex PCR (mPCR) capable of distinguishing subserotypes Ia, Ib/Ic, II, and III was also developed.

\section{Material and methods}

\section{Bacterial strains and taxonomic characterization}

A total of 73 bacterial strains of the genera Streptococcus, Lactococcus, Vagococcus, Carnobacterium, Aeromonas, Renibacterium, Tenacibaculum, Listeria, Flavobacterium, Yersinia, Pseudomonas, Vibrio, Listonella, and Edwarsiella were used in the present study (Table 1). This bacterial collection included reference strain representatives of the different serotypes described for $S$. parauberis (serotypes I, II, III. and non-typeable strains). These reference strains were used for the optimization of the qPCR and mPCR protocols and as positive control in all reactions. The bacteria were grown on Tryptic Soy Agar with $1 \% \mathrm{NaCl}(w / v)$ (TSA-1) at $25^{\circ} \mathrm{C}$ for 24 h. Tenacibaculum and Flavobacterium strains were grown at the appropriate temperatures on Flexibacter maritimus medium (FMM) (Pazos et al. 1996) and FLP medium (Cepeda et al. 2004), respectively. Stock bacterial cultures were frozen at $-80{ }^{\circ} \mathrm{C}$ in Microbank ${ }^{\mathrm{TM}}$ commercial medium (Pro-Lab Diagnostics, ON, Canada). Before the analysis, the taxonomic position of the strains used in this study was confirmed by using standard microbiological analysis and/or speciespecific PCR as described (Buller 2014; Austin and Austin 2016; Torres Corral et al. 2019). Serological typing of $S$. parauberis strains was carried out by Dot-Blot and agglutination tests as described by Torres-Corral et al. (2019), using formalin-killed cells (FKC) and rabbit whole cell antisera against the strains NCDO 2020 and SK451/04 (serotype III) obtained in our laboratory and antisera against the strains of subserotypes Ia, Ib, Ic, and serotype II, kindly provided by Dr. Kanai.

\section{Identification of the locus cps based on genomic comparisons}

Genomic comparison was performed using fifteen wholegenomes of S. parauberis and six nucleotide sequences of loci cps of S. parauberis (Supplemental Table S1). The nucleotide sequences used in this study were retrieved from the National 
Table 1 Bacterial strains used in the present study and results of serological analysis and PCR typing using the primers SP3-130F and SP3-130R

\begin{tabular}{|c|c|c|}
\hline Bacterial strains & Serotype identified & $\mathrm{mPCR} / \mathrm{qPCR}$ \\
\hline \multicolumn{3}{|l|}{ Reference strains } \\
\hline Streptococcus parauberis NCIMB 703043 & Serotype III & $+/+$ \\
\hline S. parauberis NCDO 2020 & Serotype IV & $-1-$ \\
\hline Streptococcus iniae CECT 7363 & NA & $-1-$ \\
\hline Streptococcus agalactiae DSM 28863 & NA & $-1-$ \\
\hline S. agalactiae CECT 183 & NA & $-1-$ \\
\hline Streptococcus suis CECT 958 & NA & $-1-$ \\
\hline Streptococcus mutans KCCM 40105 & NA & $-1-$ \\
\hline Streptococcus uberis CECT 994 & NA & $-1-$ \\
\hline Vagococcus salmoninarum CECT 5810 & NA & $-1-$ \\
\hline Lactococcus garvieae NCDO 2155 & NA & $-1-$ \\
\hline L. garvieae CECT 5274 & NA & $-/-$ \\
\hline Lactococcus piscium CECT 4493 & NA & $-/-$ \\
\hline Lactococcus lactis ssp. lactis KCTC 3769 & NA & $-1-$ \\
\hline \multicolumn{3}{|l|}{ Clinical isolates } \\
\hline S. parauberis, diseased turbot (Spain, $n=10$ ) & Serotype III & $+/+$ \\
\hline S. parauberis, diseased flounder (Japan, $n=3$ ) & Serotype II & $+/-$ \\
\hline S. parauberis, diseased flounder (Japan, $n=3$ ) & Serotype I & $+/-$ \\
\hline S. parauberis, diseased flounder (Japan, $n=2$ ) & Non-typeable & $+/-$ \\
\hline S. iniae, diseased marine fish (Spain, $n=5$ ) & NA & $-1-$ \\
\hline S. agalactiae, unknown (Spain, $n=3$ ) & NA & $-1-$ \\
\hline V. salmoninarum, diseased trout (Spain, $n=5$ ) & NA & $-1-$ \\
\hline L. garvieae, diseased trout (Spain, $n=5$ ) & NA & $-1-$ \\
\hline L. lactis, diseased trout (Spain, $n=2$ ) & NA & $-1-$ \\
\hline \multicolumn{3}{|l|}{ Non-related strains } \\
\hline Carnobacterium maltaromaticum ATCC 35586 & NA & $-1-$ \\
\hline Carnobacterium divergens CECT 4016 & NA & $-1-$ \\
\hline Listeria monocytogenes CECT 934 & NA & $-1-$ \\
\hline Renibacterium salmoninarum ATCC 33209 & NA & $-1-$ \\
\hline Aeromonas salmonicida ATCC 33658 & NA & $-1-$ \\
\hline A. salmonicida NCIMB 2261 & NA & $-/-$ \\
\hline A. salmonicida subsp. achromogenes CECT 895 & NA & $-/-$ \\
\hline A. salmonicida subsp. masoucida CECT 896 & NA & $-1-$ \\
\hline Aeromonas hydrophila CECT 4330 & NA & $-1-$ \\
\hline Aeromonas piscicola CECT 7443 & NA & $-1-$ \\
\hline Vibrio anguillarum ATCC 43306 & NA & $-1-$ \\
\hline Listonella pelagia NCIMB 1900 & NA & \\
\hline Tenacibaculum maritimum NCIMB 2154 & NA & $-1-$ \\
\hline Tenacibaculum dicentrarchi NCIMB 14598 & NA & $-1-$ \\
\hline Tenacibaculum gallaicum DSM 18841 & NA & $-1-$ \\
\hline Tenacibaculum soleae CECT 7292 & NA & $-1-$ \\
\hline Flavobacterium psychrophilum NCIMB 13384 & NA & $-/-$ \\
\hline Flavobacterium branchiophilum ATCC 35035 & NA & $-1-$ \\
\hline Flavobacterium plurextorum CECT 7844 & NA & $-1-$ \\
\hline Yersinia ruckeri CECT 955 & NA & $-1-$ \\
\hline Pseudomonas aeruginosa CECT 108 & NA & $-1-$ \\
\hline Escherichia coli CECT 99 & NA & $-1-$ \\
\hline
\end{tabular}

ATCC American Type Culture Collection (Maryland, USA), NCDO National Collection of Dairy Organism (Washington DC, USA), CECT Spanish Type Culture Collection (Valencia, Spain), DSM Leibniz Institute DSMZ-German Collection of Microorganisms and Cell Cultures (Braunschweig, Germany), $N C I M B$ National Collection of Industrial and Marine Bacteria (Aberdeen, UK), + typeable strains, - non-typeable strains, NA non-applicable 
Center for Biotechnology Information (NCBI) genome database. For comparative purposes, genomic sequences were annotated using the Rapid Annotations using Subsystems Technology (RAST) automated prokaryotic annotation server (Aziz et al. 2008). The accuracy of the RAST functional annotation of the draft genomes was compared with manual searches of homologous genes in the NCBI database and with the RAST annotation of the $S$. parauberis genomes classified as "complete or chromosome" by the NCBI genome database, corresponding to the reference strains KCTC 11537 and NCDFD 2020 (Supplemental Table S1), from the Korean Collection for Type Cultures (KCTC) and from National Cohort of Dairy Farms (NCDF). The capsule biosynthesis (cps) locus of the $S$. parauberis was identified on the basis of multiple nucleotide sequence alignments using Mauve Genome Alignment 2.4.0 (Darling et al. 2004) and ClustalW (Thompson et al. 1994). The genes of the cps loci were designated following the scheme proposed by Tu et al. (2015a).

\section{Sequencing of cps $3 \mathrm{~K}$ gene}

The nucleotide sequence of the loci $c p s$ of the strains KRS02083 (subserotype Ia), NUF1003 (subserotype Ib), NUF1071(subserotype Ic), NUF1032 (serotype II), 2007-1 and NUF1095 (non-typeable strains), strains isolated from turbot (AZ70.1 and T1), strains isolated from wild striped bass (RP17, PL23, PL9, RP15, RP25, N198_2, and N11), strains isolated from Japanese flounder (KCTC 11537, KRS02083, KCTC11980 and KRS02109), and strain isolated from Japanese black seaperch (SK-417) of S. parauberis were retrieved from the GenBank database (https://www.ncbi.nlm. nih.gov/), aligned and compared using the ClustalW (Thompson et al. 1994) and Nucleotide Basic Local Alignment Search Tool (BLASTn) programs to identify serotype-specific regions.

The $c p s 3 K$ gene of the variable region of the $c p s$ loci of $S$. parauberis, detected after in silico analysis of the genome of strains isolated from turbot and striped bass, was sequenced using the primers cps3K-F (5'-ACCCATGAGCTTTT TCTTCCAT-3') and cps3K-R (5'-ATGTTATGTGCATG GCTCGT-3') designed using Pick primer and PrimerBLAST tools (http://blast.ncbi.nlm.nih.gov/Blast.cgi). The primers amplify a region of $1034 \mathrm{bp}$ of the cps $3 K$ gene. Genomic DNA from three strains isolated from turbot and belonging to serotype III (NCIMB 703043, SK451/04, and SK537/10) was extracted using InstaGene matrix (BioRad, Madrid, Spain) and submitted to the Spanish Collection of Type Cultures (CECT, Valencia, Spain) for sequencing analysis using an ABI 3730xl sequencer (Applied Biosystems). The obtained sequences were cleaned and assembled using CodonCode Aligner v. 9.0.1 (CodonCode Co., USA). The partial sequences of the $\operatorname{cps} 3 K$ gene were then aligned and compared with the sequences of the loci cps of S. parauberis strains deposited in the NCBI database (Supplemental Table S1) using the ClustalW software and BLASTn to search for putative serotype III specific regions.

\section{Typing of S. parauberis using multiplex and real-time PCR}

Primer design Pick primer and Primer-BLAST tools were used to design primers specific to identify strains of $S$. parauberis of serotype III, based on a sequence of $1086 \mathrm{bp}$ of the S. parauberis pyruvyl transferase polysaccharide ( $c p s 3 K$ ) gene (GenBank accession no. NSGS0100000014.1). To evaluate primer properties and to optimize real-time PCR conditions, IDT SciTools Web (Integrated DNA Technologies, Coralville, IA, USA) was used. The forward primer SP3-130F (5'- GACCAACACCAGCA CCAATA $-3^{\prime}$, positions 738 to 757 in the $S$. parauberis cps $3 \mathrm{~K}$ gene) and the reverse primer SP3-130R (5'TTTGGACAACCTGGAAGAGC - $3^{\prime}$, positions 848 to 867 in the $S$. parauberis cps $3 K$ gene) were designed to amplify a 130 bp internal fragment of $c p s 3 K$ gene of $S$. parauberis. Primers were synthesized by IDT (Integrated DNA Technologies, Coralville, Iowa, USA). The specificity of the SP3-130F and SP3-130R primers was assessed in silico using the BLASTn tool against other sequences present in the GenBank database.

Multiplex PCR Sequences of primer sets used to identify strains of S. parauberis of the serotypes I (subtypes Ia, Ib, and Ic), II and III using the optimized mPCR assay are shown in Table 2. Amplification was performed in $50 \mu \mathrm{l}$ of reaction using Phire Hot Start II DNA polymerase (Thermo Scientific, Madrid, Spain), $2.4 \mu \mathrm{M}$ of each primer and $2 \mu \mathrm{l}$ of DNA template. The conditions of PCR amplifications were initial denaturation step of $98{ }^{\circ} \mathrm{C}$ for $10 \mathrm{~min}$, followed by 30 cycles of denaturation at $98{ }^{\circ} \mathrm{C}$ for $30 \mathrm{~s}$, annealing at $60{ }^{\circ} \mathrm{C}$ for $30 \mathrm{~s}$, extension at $72{ }^{\circ} \mathrm{C}$ for $90 \mathrm{~s}$, and a final extension at $72{ }^{\circ} \mathrm{C}$ for $5 \mathrm{~min}$. All multiplex products were checked by agarose gel electrophoresis (3\% w/v in TAE $1 \mathrm{X}$ buffer) and stained with Redsafe nucleic acid staining solution $(20,000 \times)$ (iNtRON Biotechnology, Seongnam-Si, Korea). GeneRuler 1-kb DNA ladder (Fermentas, Madrid, Spain) was used as molecular weight marker.

Real-time PCR A qPCR method was also optimized for specific detection of strains of $S$. parauberis of the serotype III in pure cultures and infected fish tissues. For this assay, bacterial suspensions containing $1 \times 10^{8} \mathrm{cell} / \mathrm{ml}$ (absorbance at $620 \mathrm{~nm}$ 0.131 ) and DNA extracted from bacterial cultures and fish tissues were used as template. Amplification was performed using Maxima SYBR Green qPCR Master Mix (2X), no ROX (Thermo Scientific, Waltham, MA, USA), $2.4 \mu \mathrm{M}$ of each primer and $1 \mu \mathrm{l}$ of template (bacterial suspensions or DNA from bacterial cultures or tissues of fish). The thermal cycling conditions comprised an initial denaturation of $5 \mathrm{~min}$ at $95^{\circ} \mathrm{C}$, 
Table 2 Primers used in this study

\begin{tabular}{lllc}
\hline Primers used for S. parauberis typing & Sequence $\left(5^{\prime} \rightarrow 3^{\prime}\right)$ & Amplicon size \\
\hline Serotype I & For-Ia & ATTGTTAGTCATTCAGTTGT & $213 \mathrm{bp}$ \\
& Rev-Ia & AATTATAGTCAACAGTCCAG & \\
& For-Ib/Ic & ATTTCTACCAGGTTACTTTG & $303 \mathrm{bp}$ \\
& Rev-Ib/Ic & ACATCTCGAAACTTCATATT & \\
Serotype II & For-II & GAACTACTTAGGTTTAGCAT & $413 \mathrm{bp}$ \\
& Rev-II & ACTTGTAAATAGGATTGCT & \\
Serotype III & SP3-130F & GACCAACACCAGCACCAATA & $130 \mathrm{bp}$ \\
& SP3-130R & TTTGGACAACCTGGAAGAGC & \\
\hline
\end{tabular}

followed by 30 cycles of denaturation at $95{ }^{\circ} \mathrm{C}$ for $30 \mathrm{~s}$ and annealing at $60{ }^{\circ} \mathrm{C}$ for $10-30 \mathrm{~s}$. For bacterial suspensions, the initial denaturation step was increased to $10 \mathrm{~min}$. A melt curve analysis was performed after the last cycle using a temperature gradient from 65 to $95^{\circ} \mathrm{C}$ and a ramp speed of $0.5^{\circ} \mathrm{C} \mathrm{s}^{-1}$ (for $10 \mathrm{~s}$ ) with continuous fluorescence data measurement. DNA isolated from pure cultures of the reference strain S. parauberis NCIMB 703043, which belongs to serotype III, was used as a positive control in $\mathrm{qPCR}$ reactions. Reactions lacking DNA template (no template control) and reactions containing DNA from non-infected tissues were used as negative controls. All qPCR assays were performed in triplicate. The expected size of the amplicons was confirmed by gel electrophoresis as described above.

The specificity in vitro of SP3-130F and SP3-130R primers was tested using multiplex and real-time PCR assays. In PCR assays, the existence of cross-amplifications was evaluated using as template bacterial cells and DNA from strains of serotype III of $S$. parauberis ( $n=11$ strains) and non-target bacteria (Table 1).

The sensitivity of the SP3-130F and SP3-130R primers was evaluated using amplification products of the reference strain NCIMB 703043 and a clinical isolate of $S$. parauberis serotype III. The amplification products were purified using the PureLink® PCR (Invitrogen, Barcelona, Spain) purification kit and quantified using Qubit ${ }^{\circledR} 2.0$ and the Qubit ${ }^{\circledR}$ DNA HS Assay Kit (Invitrogen, Barcelona, Spain). Dilutions of the purified amplification product were used to generate a linear standard curve by plotting the $\mathrm{Cq}$ values obtained for each decimal amplicon dilution against the logarithm of the amount (ng) of the amplicon. Each amplicon dilution was tested in triplicate. The copy number of the amplification products was quantified as indicated by the IDT SciTools Web Tools (Integrated DNA Technologies, Coralville, IA, USA) using the formula:

Number of copies (molecules)

$$
=\frac{X n g * 6.0221 \times 10^{23} \text { molecules } / \mathrm{mol}}{\left(\boldsymbol{N} * 660 \frac{\mathrm{g}}{\mathrm{mol}}\right) * 1 \times 10^{9} \mathrm{ng} / \mathrm{g}}
$$

where $\boldsymbol{X}$ is the amount of amplicon (ng), $\boldsymbol{N}$ is the length of dsDNA amplicon, and $660 \mathrm{~g} / \mathrm{mol}$ is the average mass of $1 \mathrm{bp}$ of dsDNA.

The linear standard curve was used to convert the $\mathrm{Cq}$ values obtained from the analysis of a sample into the copy number of the amplified product. Each decimal dilution of amplification products used to generate the linear standard curve by qPCR, were also analyzed by gel electrophoresis ( $2 \% w / v$ in TAE $1 \times$ buffer) to determine the analytical sensitivity of the gel electrophoresis correlated with the qPCR. The efficiency of the qPCR assay was calculated from the slope (m) of the line according to the eq. $E=10^{(-1 / \mathrm{m})}-1$.

Repeatability and reproducibility were evaluated by calculating the coefficient of variation $(\% \mathrm{CV})$ of replicates performed in the same sensitivity test (intra-assay) and of replicates performed in three independent sensitivity tests (interassay), respectively. For each test, three replicates were performed and only the results from concentrations that tested positive in all the three replicates were analyzed.

\section{qPCR detection of S. parauberis serotype III in fish sample}

The usefulness of primers (SP3-130F and SP3-130R) and qPCR protocol designed in this study for the detection and quantification of $S$. parauberis serotype III in tissues of fish inoculated with the bacterium was evaluated. Fish samples of kidney, spleen, and blood were homogenized at a concentration of $25 \%$ in saline $(0.9 \% \mathrm{NaCl})$, seeded and incubated for $1 \mathrm{~h}$ with known amounts of bacterial suspension of NCIMB 703043 strain, resulting in final concentrations $5 \times 10^{7}$ to $5 \times$ $10^{0}$ cells $/ \mathrm{ml}$ in the extract. To confirm the specificity of the qPCR protocol for detection of $S$. parauberis serotype III in fish samples, tissues samples were also artificially infected with strains representative of the other serotypes described in S. parauberis (Ia, strain KRS02083; Ib, strain NUF1003; Ic, strain NUF1071; and II, strain NUF1032). DNA from control (non-inoculated) and artificially infected fish tissues were obtained using Dynabeads DNA Direct ${ }^{\mathrm{TM}}$ (Thermo Fisher Scientific, Madrid, Spain) following manufacturer's instructions. 
The sensitivity and specificity of the qPCR was also evaluated using tissue samples (available in the laboratory), obtained from turbot experimentally infected or suffering from streptococcosis caused by $S$. parauberis serotype III. To exclude the possibility of non-specific amplifications, tissue samples from fish experimentally or naturally infected with other $S$. parauberis serotypes and other pathogens (Streptococcus iniae, Vagococcus salmoninarum, Yersinia ruckerii, L. garvieae, F. psychrophylum, and Aeromonas salmonicida) were tested in parallel. In these trials, tissue samples from healthy fish were used as negative controls. To control the quality of DNA obtained from the tissues, all fish samples were also analyzed using the primers $\beta$-actin-Fw (5'CTGAAGTACCCCATTGAGCAT- $\left.3^{\prime}\right)$ and $\beta$-actin-Rv ( $5^{\prime}$ CATCTTCTCCGTGCTT-3') which amplify a 125 bp fragment of the $\beta$-actin gene from turbot (Fernandez-Álvarez et al. 2019). The standard curve previously performed and the $\mathrm{Cq}$ value obtained in those assays were used to calculate the amount of $S$. parauberis serotype III DNA in fish samples and thereby calculate the number of copies of the $c p s 3 K$ single copy gene in that sample.

\section{Nucleotide sequence accession number}

The partial sequences of the $c p s 3 \mathrm{~K}$ gene sequenced in this study have been deposited in the NCBI (Genbank) database under the accession numbers of MT040616 (strain NCIMB 703043), MT040617 (strain SK451/04), and MT040618 (strain SK537/10).

\section{Results}

\section{General features of the cps loci of strains isolated from marine fish and mastitis}

The genomes available at NCBI of strains isolated from marine fish (AZ70.1, T1, RP17, PL23, PL9, RP15, RP25, N198_2, N11, KCTC 11537 SK-417, KRS02083, KCTC1 1980, and KRS02109) and from mastitis (NCDFD 2020) were compared with the sequences of the loci cps of strains of the serotypes Ia (strain KRS02083, sequence accession no. LC060252), Ib (strain NUF1003, LC060253), Ic (strain NUF1071, LC060254), and II (strain NUF1032, LC060255) of $S$. parauberis previously described by $\mathrm{Tu}$ et al. (2015a). The locus cps detected in these genomes has the cassette-like structure previously described for serotypes I and II (Tu et al. 2015a), in which serotype-specific genes are flanked by conserved regions. The upper conserved region of the loci $c p s$ involves five regulatory genes, lysR (homology between serotypes $>99 \%$ ) and $c p s A B C D$ (homology between serotypes $>95 \%$ ), and a processing gene, $c p s E$ (homology between serotypes $>98 \%$ ) (Supplemental Table S2, Fig. 1).
The lower conserved region involves two genes, the $c p s Q$ gene, which encodes for a dehydrogenase responsible for capsule processing (homology between serotypes $>80 \%$ ), and the $\operatorname{cps} R$ gene, which encodes for a hypothetical protein (homology between serotypes $>99 \%$ ) (Supplemental Table S2, Fig. 1). Based on the comparative genomic analysis, the variable regions of the $c p s$ loci previously associated (Tu et al. 2015a) with the serotypes Ia (strain KRS02083), Ib (NUF1003), Ic (NUF1071), and II (NUF1032) were clearly evidenced. The serotype-specific genes described for subserotype Ia (Tu et al. 2015a) were also identified in the whole genomes available in the NCBI database of strains isolated from Japanese flounder (KRS02083 and KCTC11980), suggesting that these strains were subserotype Ia. The serotype-specific region associated to serotype II was detected only in the whole genome of one strain isolated from Japanese flounder (KRS02109), suggesting that this strain was serotype II. Three variable regions (named as III, IV, and V), different to those described by Tu et al. (2015a) for subserotypes Ia, Ib, Ic, and serotype II, were also identified and represented in Fig. 1. The variable region III was detected in the genome of strains AZ70.1 (Supplemental Table S1 for NCBI accession no.) and $\mathrm{T} 1$ (Supplemental Table S1) isolated from turbot in Spain and in the genome of strains RP17, PL23, PL9, RP15, RP25, N198_2, and N11 (Supplemental Table S1) isolated from wild striped bass in USA. In this region, genes that encode transferases and epimerases were mostly detected (Supplemental Table S2 and Fig. 1). The cps $3 F$ gene of the region III, coding for a glucosyltransferase, showed homology of 99,44 , and $25 \%$ with the genes cpslaF (serotype Ia), cps $1 b G$ (serotype Ib), and cps4G (strain NCDFD2020), respectively. The genes cps $3 G$ and cps $3 P$, both epimerases encoders, showed a homology of $75 \%$ with cps $1 a G$ (serotype Ia) and of $74 \%$ with cps $1 a P$ (serotype Ia) and cps $1 b P$ (serotype Ib), respectively (Fig. 1). The genes cps $3 H$, cps $3 I$, cps $3 J$, $\operatorname{cps} 3 K$, and $\operatorname{cps} 3 L$ were only detected in the variable region III. The variable region IV that contains mainly genes encoding for transferases and dehydrogenases (Supplemental Table S2 and Fig. 1) was identified in the genome sequence (Supplemental Table S1) of the reference strain NCDO 2020 isolated from mastitis sample milk The cps $4 G$ gene, coding for transferase, showed a homology $>25 \%$ with the genes cps $1 a F$ (serotype Ia), cps $1 b G$ (serotype Ib), and $c p s 3 F$ (strains AZ70.1, T1, RP17, PL23, PL9, RP15, RP25, N198_2, and N11) (Fig. 1). The genes cps $4 F$, cps $4 H, c p s 4 I, c p s 4 J, c p s 4 k$, cps $4 L$, cps $4 M$, cps $4 N$, and cps $4 O$ did not show homology with the genes of the other variable regions. The variable region $\mathrm{V}$ was identified in the genome of the strain KCTC 11537 (Supplemental Table S1) isolated from Japanese flounder and in the genome of strain SK-417 (Supplemental Table S1) isolated from Japanese black seaperch. No homology with the genes of the other variable regions was observed. The variable regions IV and V identified in the present study 


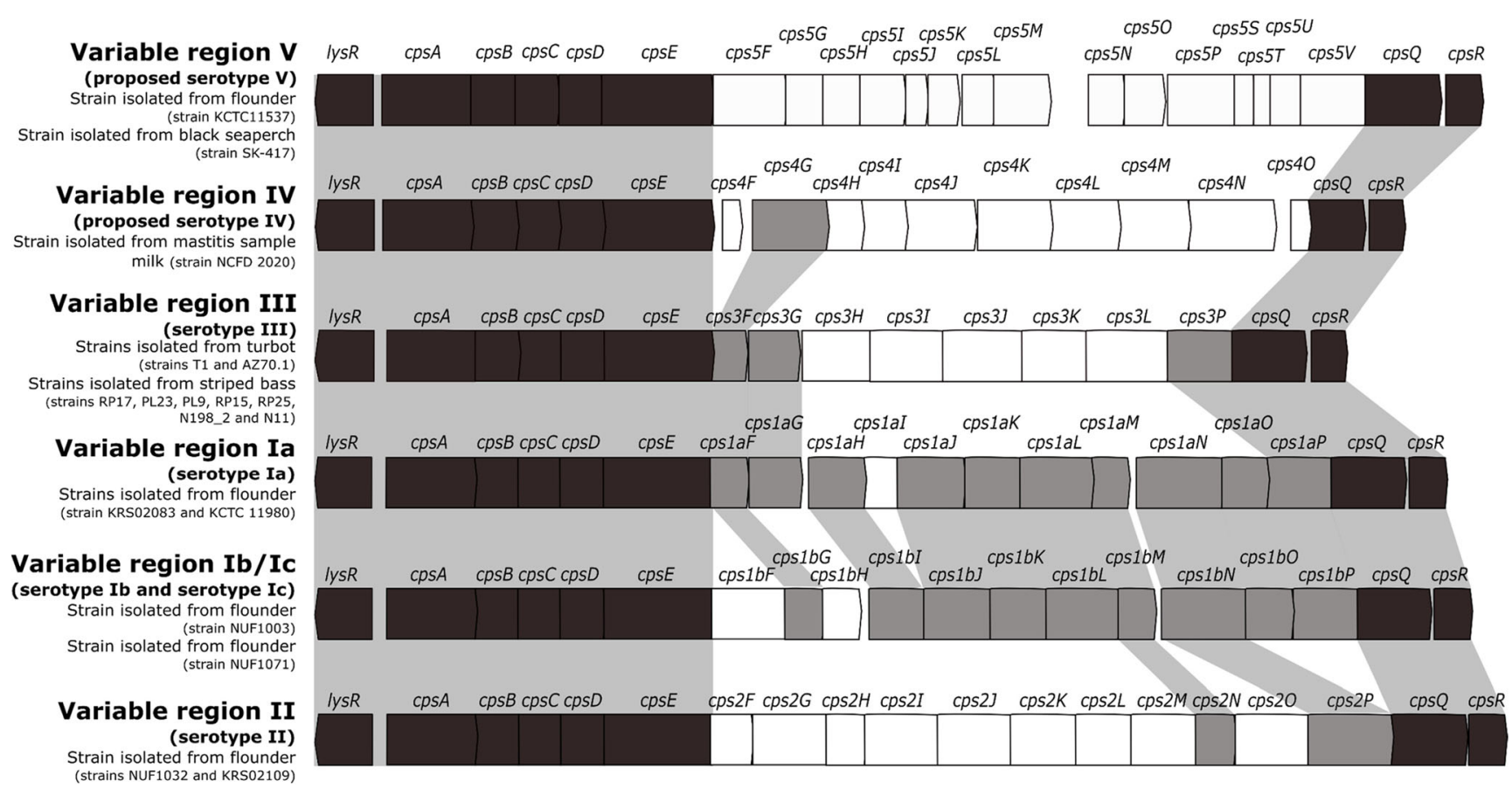

Fig. 1 Structure of loci cps of S. parauberis detected in this study. In black are represented conserved regions of the cps locus of S. parauberis, in gray are represented genes that are present in two or more serotypes, in white are represented serotype-specific regions

could represent serotype-specific regions of the new serotypes IV (strain representative NCDFD2020) and V (strains representatives KCTC 11537 and SK-417).

To investigate if the gene cps $3 K$ of the variable region III, encoding the polysaccharide pyruvyl transferase family protein, was related with the serotype III, partial sequencing and BLAST analysis was carried out using DNA from the strains of $S$. parauberis serotype III isolated from turbot NCIMB 703043 (NCBI accession number MT040616), SK451/04 (NCBI accession number MT040617), and SK537/10 (NCBI accession number MT040618).The analysis of the partial sequences obtained revealed similarities greater than $99 \%$ with the gene $\operatorname{cps} 3 K$ of the variable region III of the loci cps of the strains AZ70.1, T1, RP17, PL23, PL9, RP15, RP25, N198_2, and N11 deposited in the NCBI database. This result suggests that strains AZ70.1, T1, RP17, PL23, PL9, RP15, RP25, N198_2, and N11 could belong to serotype III of S. parauberis (Fig. 1).

\section{Typing of S. parauberis using serological tests and multiplex and real time PCR}

Serological test allowed to assign the $S$. parauberis strains tested to the serotypes I (15\% of strains), serotype II (15\%) and serotype III (55\% of strains). The strain NCDO 2020 only reacted with the homologous antiserum and was assigned to the new proposed serotype IV. Two strains did not react with the antisera tested and were designated as non-typeable (Table 1).
For primer design, the gene $\operatorname{cps} 3 K$ present in the variable region III of the locus cps detected in genomes of strains isolated from turbot (AZ70.1 and T1) and wild striped bass (strains RP17, PL23, PL9, RP15, RP25, N198_2, and N11) as well as in the genome of the strains NCIMB 703043, SK451/04, and SK537/10 was selected as target. In silico and in vitro analysis revealed that the primer pair designed in this study (SP3-130F and SP3-130R) for identifying strains of the serotype III of $S$. parauberis was specific (Table 1). Eleven strains of S. parauberis serotype III tested in this study, including the reference strain NCIMB 703043 and clinical strains isolated from diseased turbot, were positively identified by PCR at the optimal annealing temperature of $60{ }^{\circ} \mathrm{C}$. No amplifications were found when bacterial suspensions or extracted DNA from strains of other serotypes of $S$. parauberis $(n=9)$ or other no-target bacteria $(n=53)$ were used (Table 1).

Multiplex PCR allowed the typing of $S$. parauberis strains belonging to subserotype Ia (specific band of $213 \mathrm{bp}$ ), serotype II (413 bp), and serotype III (130 bp) (Fig. 2). Nontypeable strains were also detected. The mPCR assay do not allow to differentiate subserotypes Ib and Ic, which showed a characteristic band of $303 \mathrm{bp}$ (Fig. 2). No amplification products were observed using water as a negative control, DNA extracted from reference strain of $S$. parauberis NCDO 2020, or DNA extracted from other unrelated bacteria. Therefore, the results of molecular serotyping using the MPCR assay were comparable with the results obtained by serological tests (Table 1). 
Fig. 2 Multiplex PCR products of S. parauberis strains. Lanes: MM, Generuler DNA ladder $1 \mathrm{~kb}$ (Fermentas); 1, (subserotype Ia); 2, subserotype Ib; 3 , subserotype Ic; 4, serotype II; 5, serotype III; 6 and 7 , non-typeable strains; 8 , NCDO2020 (serotype IV)

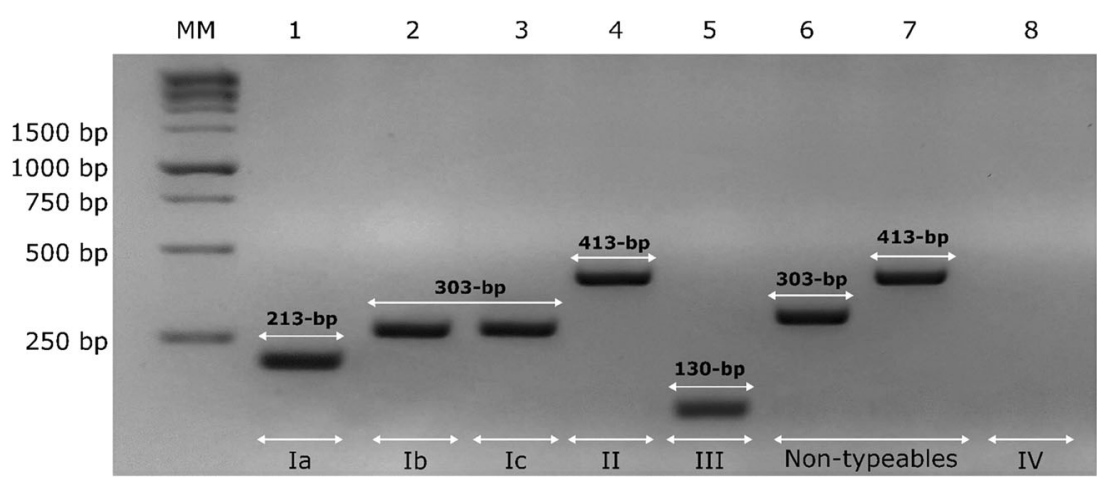

Typing of $S$. parauberis serotype III was also performed by qPCR using bacterial suspensions or DNA extracted from bacterial cultures or tissues from fish infected with strains of S. parauberis serotype III as template. A specific peak with a melting temperature (Tm) of $73 \pm 0.33{ }^{\circ} \mathrm{C}$ (Fig. 3) was obtained with DNA samples or bacterial suspensions from strains of $S$. parauberis of serotype III. No amplifications were observed using bacterial suspensions or DNA extracted from other serotypes of $S$. parauberis or unrelated bacteria (Fig. 3, Table 1). On the standard curve, regression coefficients $R^{2}=0.997$ $(\mathrm{m}=-3.3593$; efficiency $98.5 \%)$ were obtained using the DNA of the reference strain NCIMB 703043 (Fig. 4a) and $R^{2}=0.994(\mathrm{~m}=-3.3939$; efficiency $97.08 \%)$ using the DNA of a clinical isolate (strain SK 451/04) of the serotype III of $S$. parauberis (Fig. 4a). The limit of detection of the assay was $2.67 \times 10^{2}$ amplicon copies per assay (equivalent to $3.8 \times 10^{-9} \mathrm{ng}^{-1} \mathrm{l}^{-1}$ ) (Table 3 , Fig. 4a) using as template the purified amplicon of both strains. The amplification products obtained after the qPCR sensitivity test were electrophoresed at $2 \%$ agarose gel (Fig. 4b). The intra-assay \% CV values ranged from 0.17 to 7.53 (Table 3) and inter-assay \% CV values ranged from 0.50 to 6.33 (Table 3 ).

\section{Applicability of the qPCR assay to detect S. parauberis serotype III in fish samples}

Detection levels of $4.52 \times 10^{8}$ to $1.76 \times 10^{2}$ copies of the $\operatorname{cps} 3 \mathrm{~K}$ gene per $\mu \mathrm{l}$ were obtained using qPCR protocol and samples generated in the laboratory (extracted DNA, bacterial suspensions or artificially infected fish tissues) as template (Table 4). The bacterial load detected in tissues from diseased turbot with clinical signs of streptococcosis ranged from $1.25 \times 10^{4}$ to $3.02 \times 10^{2}$ copies of the cps $3 K$ gene $(\mathrm{Cq}$ values ranging from 21.58 to 27.02). Positive amplifications were also obtained using as template DNA extracted from tissues of fish experimentally infected without clinical signs of streptococcosis, detecting from $1.07 \times 10^{3}$ to $1.76 \times 10^{2}$ copies of the cps $3 K$ gene $(\mathrm{Cq}$ values ranging from 25.17 to 27.82). No amplifications were observed using as template DNA extracted from tissue samples from healthy fish, from fish infected with $S$. parauberis of other serotypes or with other bacterial pathogen. The $\beta$-actin gene was detected in all fish tissue samples, discarding false negative results.
Fig. 3 Melting curve analysis using qPCR and SP3-130F and SP3-130R primers, showing a specific melting peak at $\operatorname{Tm} 73 \pm$ $0.40{ }^{\circ} \mathrm{C}$ using bacterial DNA from strains of Streptococcus parauberis serotype III ( $n=11$ strains) and the lack of amplification obtained using DNA from strains of other serotypes of $S$. parauberis or unrelated bacteria and negative control

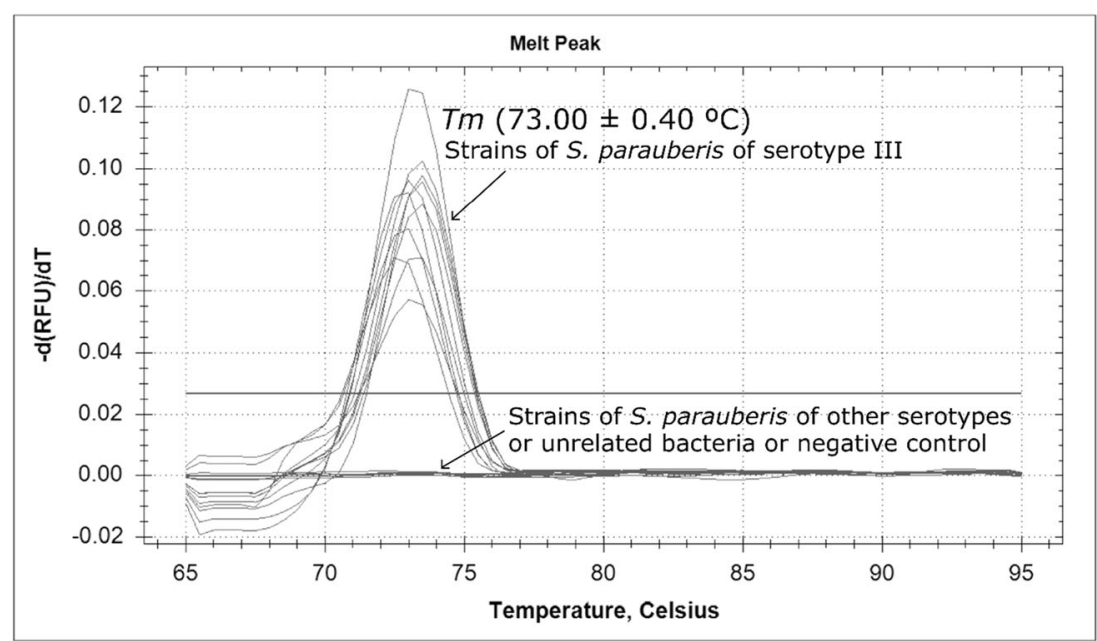


(a)

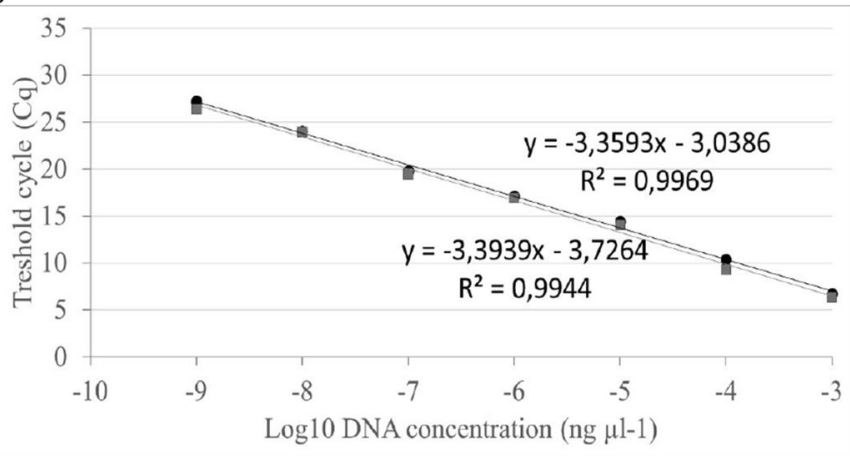

Fig. 4 Standard curves obtained by qPCR from the amplification of 10fold dilutions of purified amplicon obtained using SP3-130F and SP3$130 \mathrm{R}$ primers and DNA from the reference strain $S$. parauberis NCIMB 703043 (black circles) and a clinical isolate of S. parauberis (SK451/04) (gray squares) (a) and the corresponding electrophoresis gel showing the

\section{Discussion}

Accurate and rapid determination of the $S$. parauberis serotype is of crucial importance for surveillance studies and vaccine development. S. parauberis was originally described as phenotypically and serologically homogeneous (Toranzo et al. 1995), but several serological types have been reported over the years using conventional serotyping methods (Kanai et al. 2009, 2015; Torres Corral et al. 2019). Conventional serotyping based on the use of mono- or polyclonal sera and inactivated whole cell is the method more commonly used in clinical diagnostic laboratories. However, the use of this method is limited by the unavailability of specific commercial sera which difficult the comparison of the results within laboratories. PCR-based bacterial serotyping protocols have therefore emerged as a promising alternative to conventional serotyping. These mechanisms do not require the use of sera, but it is necessary to identify molecular markers that determine serotypes as a prerequisite for designing specific primers (Fratamico et al. 2016; Rochat et al. 2017).

In order to identify a molecular marker determining S. parauberis serotype III, whole genome of 15 strains of (b)

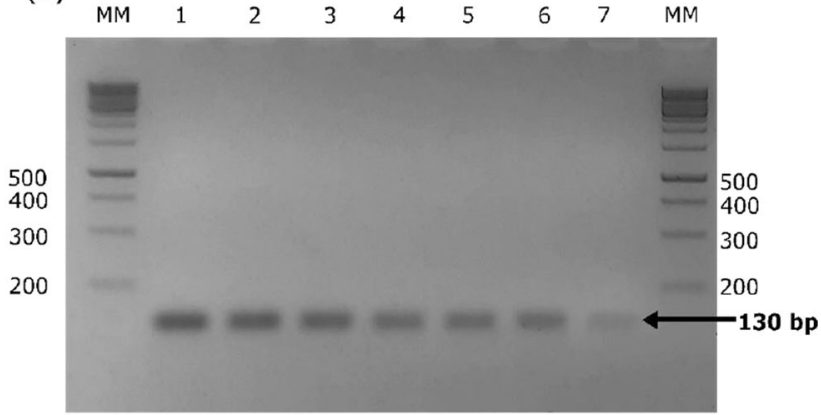

band intensities correlating with the number of copies of the cps $3 \mathrm{~K}$ gene (b). Lanes: MM, Generuler DNA ladder 1-kb (Fermentas); 1, $2.67 \times 10^{8}$ copies; $2,2.67 \times 10^{7}$ copies; $3,2.67 \times 10^{6}$ copies; $4,2.67 \times 10^{5}$ copies; 5 , $2.67 \times 10^{4}$ copies; $6,2.67 \times 10^{3}$ copies; $7,2.67 \times 10^{2}$ copies

S. parauberis isolated from different hosts and geographical origins (Nho et al. 2011. 2013; Park et al. 2013; Oguro et al. 2014; Haines et al. 2016), were compared with the sequences of the loci cps described by Tu et al. (2015a) for serotypes I and II. A locus cps with a cassette-like structure, a variable region flanked by conserved regions, was found in 15 genomes of $S$. parauberis analyzed in the present study. This locus structure was previously described in the cps loci of $S$. parauberis strains belonging to serotypes I and II (Tu et al. 2015b), and other Streptococcus (Bentley et al. 2006; Jiang et al. 2006; Wang et al. 2011; Morais et al. 2018). In addition, in the present study, three variable regions (named III, IV, and V) within the loci cps, different from those described for serotypes I and II, were identified. These variable regions could represent serotype-specific regions of three different serotypes (serotypes III, IV, and V) of S. parauberis. In silico comparative analysis of the partial sequence of the cps $3 K$ gene of the variable region III obtained from three S. parauberis strains of the serotype III showed more than 99\% homology with the variable region III detected in the genome of $S$. parauberis available in the NCBI database and less than $40 \%$ homology with genes encoding the same
Table 3 Determination of $\mathrm{C}_{\mathrm{q}}$ values obtained from the amplification of tenfold dilutions of a purified amplicon of the cps $3 K$ gene of the strain NCIMB 703043 obtained by qPCR

\begin{tabular}{|c|c|c|c|c|c|c|}
\hline \multirow[b]{2}{*}{$\begin{array}{l}\text { DNA concentration } \\
(\mathrm{ng} / \mu \mathrm{l})\end{array}$} & \multicolumn{3}{|c|}{ Intra-assay variance } & \multicolumn{3}{|c|}{ Inter-assay variance } \\
\hline & $\mathrm{C}_{\mathrm{q}}$ values & $\begin{array}{l}\text { No. of target } \\
\text { copies }\end{array}$ & $\% \mathrm{CV}$ & $\mathrm{C}_{\mathrm{q}}$ values & $\begin{array}{l}\text { No. of target } \\
\text { copies }\end{array}$ & $\% \mathrm{CV}$ \\
\hline $3.8 \times 10^{-3}$ & $6.77 \pm 0.51$ & $2.67 \times 10^{8}$ & 7.53 & $6.48 \pm 0.41$ & $2.67 \times 10^{8}$ & 6.33 \\
\hline $3.8 \times 10^{-4}$ & $10.40 \pm 0.10$ & $2.67 \times 10^{7}$ & 0.96 & $10.71 \pm 0.44$ & $2.67 \times 10^{7}$ & 4.09 \\
\hline $3.8 \times 10^{-5}$ & $14.43 \pm 0.08$ & $2.67 \times 10^{6}$ & 0.55 & $13.97 \pm 0.65$ & $2.67 \times 10^{6}$ & 4.66 \\
\hline $3.8 \times 10^{-6}$ & $17.11 \pm 0.21$ & $2.67 \times 10^{5}$ & 1.23 & $16.98 \pm 0.19$ & $2.67 \times 10^{5}$ & 1.12 \\
\hline $3.8 \times 10^{-7}$ & $19.83 \pm 0.06$ & $2.67 \times 10^{4}$ & 0.30 & $19.90 \pm 0.10$ & $2.67 \times 10^{4}$ & 0.50 \\
\hline $3.8 \times 10^{-8}$ & $24.07 \pm 0.04$ & $2.67 \times 10^{3}$ & 0.17 & $23.80 \pm 0.39$ & $2.67 \times 10^{3}$ & 1.63 \\
\hline $3.8 \times 10^{-9}$ & $27.21 \pm 0.11$ & $2.67 \times 10^{2}$ & 0.40 & $27.13 \pm 0.12$ & $2.67 \times 10^{2}$ & 0.44 \\
\hline
\end{tabular}


Table 4 Samples tested by qPCR for the detection of $S$. parauberis serotype III

\begin{tabular}{llll}
\hline Sample & Concentration range & Results $\left(\mathrm{C}_{\mathrm{q}}\right.$ ranges $)$ & No. of target copies \\
\hline Genomic DNA & $3.8 \times 10^{-3}-3.8 \times 10^{-9}(\mathrm{ng} / \mu \mathrm{l})$ & $+(6.27-27.21)$ & $4.52 \times 10^{8}-2.67 \times 10^{2}$ \\
Bacterial & $1.0 \times 10^{8}-1.0 \times 10^{1}(\mathrm{CFU} / \mathrm{ml})$ & $+(10.36-27.55)$ & $2.74 \times 10^{7}-2.11 \times 10^{2}$ \\
Kidney and spleen & $5 \times 10^{7}-5 \times 10^{3}(\mathrm{CFU} / \mathrm{ml})$ & $+(14.71-27.81)$ & $1.39 \times 10^{6}-1.76 \times 10^{2}$ \\
Blood & $5 \times 10^{7}-5 \times 10^{5}(\mathrm{CFU} / \mathrm{ml})$ & $+(23.94-25.71)$ & $2.49 \times 10^{3}-7.37 \times 10^{2}$ \\
\hline
\end{tabular}

protein in other bacterial species. These results indicated that cps $3 \mathrm{~K}$ gene is specific of the serotype III strains and suggest that strains isolated from turbot in Spain and striped bass in USA, which genomes are deposited in the NCBI database, could be included into the serotype III of $S$. parauberis. Based on these results cps $3 K$ gene constitutes an excellent target for design of primers for the detection of strains of $S$. parauberis serotype III, thereby decreasing the possibility of false positive results. Thus, a primers pair SP3-130F and SP3-130R were designed based on the sequence of the cps $3 K$ gene for rapid and specific detection of strains of S. parauberis serotype III using PCR assays. Multiplex PCR assay was optimized for the detection and differentiation of the main serotypes of $S$. parauberis causing disease in fish (serotypes I, II and III) using the primers described by Tu et al. (2015b) and the primers SP3-130F and SP3-130R designed in the present study. The high correspondence observed between the results of conventional serotyping using antisera and molecular serotyping using multiplex PCR suggests that this rapid and specific typing method could be used in clinical diagnostic laboratories and/or epidemiological studies.

Typing of strains of $S$. parauberis of serotype III was also performed by qPCR using bacterial suspensions or DNA extracted from bacterial cultures or tissues from fish infected with strains of $S$. parauberis serotype III as template. PCR analysis demonstrated $100 \%$ specificity, since only DNA from strains of $S$. parauberis serotype III were amplified giving a specific peak at a $\mathrm{Tm}$ of $73 \pm 0.33^{\circ} \mathrm{C}$. Similar Tm value was obtained using as template DNA from fish tissues experimentally or naturally infected with $S$. parauberis of serotype III. No amplifications were observed using bacterial suspensions or DNA extracted from other serotypes of $S$. parauberis or unrelated bacteria. The quantification of the bacterial load was assessed through a standard curve generated by plotting the values of $\mathrm{Cq}$ versus the logarithm of the amount (ng) of amplicon as previously described (Fernández-Álvarez et al. 2016; Torres-Corral et al. 2019). A detection limit of $2.67 \times$ $10^{2}$ copies of the amplicon per $\mu$ l (equivalent to $3.8 \times 10^{-9} \mathrm{ng}$ / $\mu$ of genomic DNA) was obtained using the designed qPCR procedure.

The applicability of the qPCR protocol and SP3-130F and SP3-130R primers designed was tested using lethal (kidney and spleen) and non-lethal (blood) fish samples. High specificity and sensitivity were found using the described protocol and lethal fish samples, with detection levels of $1.25 \times$ $10^{4}$ to $1.76 \times 10^{2}$ gene copies of the $c p s 3 \mathrm{~K}$ gene in tissues of fish infected with strains of $S$. parauberis belonging to serotype III. High levels of detection $\left(2.49 \times 10^{3}-7.37 \times 10^{2}\right.$ gene copies) were also observed using this qPCR protocol and DNA obtained from non-lethal fish samples (blood samples) as template, suggesting that this non-lethal diagnostic and typing method could be used without the need for bacterial isolation or the use of anti-S. parauberis sera. Detection levels using DNA extracted from infected fish tissues were lower than those obtained from pure bacterial cultures (bacterial suspensions or DNA extracted from bacterial cultures), possibly due to the presence of host DNA or tissue inhibitors such as hemoglobin or serum proteins (Wiklund et al. 2000; Cepeda et al. 2003; Fernández-Álvarez et al. 2019; Torres-Corral et al. 2019). Further studies using a high number of strains isolated from different fish species and other animals in different geographic area will help to determine the serological diversity of $S$. parauberis and to develop new diagnostic tools.

In conclusion, this study describes the predicted structure of the cps loci involved in CPS biosynthesis for S. parauberis of the serotypes III, IV, and V, suggesting the existence of new serotypes within this species. Serotype-specific regions were used to optimize a mPCR protocol capable of differentiating the main $S$. parauberis serotypes causing disease in fish (serotypes I, II, and III). In addition, a qPCR assay to identify and quantify strains of $S$. parauberis belonging to serotype III from bacterial cultures and fish tissues has been optimized. These PCR protocols could be useful tool in epidemiological surveillance studies or for diagnostic purposes using bacterial culture and lethal and non-lethal fish tissues as samples. The PCR methods described are inexpensive with low risk of contamination that can be implemented as a tool for diagnosis and typing in clinical laboratories, thus avoiding the disadvantages of conventional serological methods.

Acknowledgments This work was partially supported by the Proof of Concept Program "Acelerador de Transferencia" from the Universidade of Santiago de Compostela (Spain). Yolanda Torres Corral was supported by a grant from the Universidade of Santiago de Compostela under the program "Acelerador de Transferencia". The authors are grateful to Dr. Kanai for supply the antisera against serotype I (subtypes Ia, Ib, I c) and II of $S$. parauberis and their representative strains and to Álvaro Robles, from the Aquaculture Division of Isidro 1952 SL, for supplying the fish used in this study. 
Authors' contributions All authors contributed to the study conception and design. Material preparation, data collection, and analysis were performed by Yolanda Torres-Corral and Y sabel Santos. The first draft of the manuscript was written by Yolanda Torres-Corral and all authors commented on previous versions of the manuscript. All authors read and approved the final manuscript.

Funding information This work was partially supported by the Proof of Concept Program "Acelerador de Transferencia" from the Universidade of Santiago de Compostela (Spain).

Availability of data and material The dataset used and/or analyzed during the current study are available from the corresponding author on reasonable request.

\section{Compliance with ethical standards}

Conflict of interest The authors declare that they have no conflict of interest.

Ethics approval The studies presented in this manuscript were approved by the USC Bioethics Committee.

\section{References}

Aoki T, Takami K, Kitao T (1990) Drug resistance in a non-hemolytic Streptococcus sp. isolated from cultured yellowtail Seriola quinqueradia. Dis Aquat Org 8:171-177

Austin B, Austin DA (2016) Bacterial fish pathogens, Sixth Edit. Springer, Chichester

Aziz RK, Bartels D, Best AA, DeJongh M, Disz T, Edwards RA, Formsma K, Gerdes S, Glass EM, Kubal M, Meyer F, Olsen GJ, Olson R, Osterman AL, Overbeek RA, McNeil LK, Paarmann D, Paczian T, Parrello B, Pusch GD, Reich C, Stevens R, Vassieva O, Vonstein V, Wilke A, Zagnitko O (2008) The RAST Server: rapid annotations using subsystems technology. BMC Genomics 9:75. https://doi.org/10.1186/1471-2164-9-75

Baeck GW, Kim JH, Gomez DK, Park SC (2006) Isolation and characterization of Streptococcus sp. from diseased flounder (Paralichthys olivaceus) in Jeju Island. J Vet Sci 7:53-58

Bentley SD, Aanensen DM, Mavroidi A, Saunders D, Rabbinowitsch E, Collins M, Donohoe K, Harris D, Murphy L, Quail MA, Samuel G, Skovsted IC, Kaltoft MS, Barrell B, Reeves PR, Parkhill J, Spratt BG (2006) Genetic analysis of the capsular biosynthetic locus from all 90 pneumococcal serotypes. PLoS Genet 2:e31. https://doi.org/ 10.1371/journal.pgen.0020031

Buller N (2014) Bacteria and fungi from fish and other aquatic animals, 2nd edit. CABI Publishing, Bentley

Cepeda, García-Márquez S, Santos Y (2003) Detection of Flexibacter maritimus in fish tissue using nested PCR amplification. J Fish Dis 26:65-70

Cepeda C, García-Márquez S, Santos Y (2004) Improved growth of Flavobacterium psychrophilum using a new culture medium. Aquaculture 238:75-82. https://doi.org/10.1016/j.aquaculture. 2004.05.013

Cho MY, Il LJ, Kim MS, Choi HJ, Lee DC, Kim JW (2008) Isolation of Streptococcus parauberis from starry flounder, Platichthys stellatus Pallas. J Fish Pathol 21:209-2017

Darling ACE, Mau B, Blattner FR, Perna NT (2004) Mauve: multiple alignment of conserved genomic sequence with rearrangements. Genome Res 14:1394-1403. https://doi.org/10.1101/gr.2289704. tion
Demczuk W, Martin I, Mulvey M, Mauffrey F, Bekal S, Domingo M, Martineau C, Le S, Doualla-bell F, Longtin J, Lefebvre B (2017) Comparison of sequential multiplex PCR, sequetyping and whole genome sequencing for serotyping of Streptococcus pneumoniae. PLoS One 12:1-16

Domenech A, Fernandez Garayzabal JF, Pascual C, Garcia JA, Cutuli MT, Moreno MA, Collins MD, Dominguez L (1996) Streptococcosis in cultured turbot, Scophthalmus maximus (L), associated with Streptococcus parauberis. J Fish Dis 19:33-38. https://doi.org/10.1111/j.1365-2761.1996.tb00117.x

Fernández-Álvarez C, González SF, Santos Y (2016) Development of a SYBR green I real-time PCR assay for specific identification of the fish pathogen Aeromonas salmonicida subspecies salmonicida. Appl Microbiol Biotechnol 100:10585-10595. https://doi.org/10. 1007/s00253-016-7929-2

Fernández-Álvarez C, González SF, Santos Y (2019) Quantitative PCR coupled with melting curve analysis for rapid detection and quantification of Tenacibaculum maritimum in fish and environmental samples. Aquaculture 498:289-296. https://doi.org/10.1016/j. aquaculture.2018.08.039

Fratamico PM, Debroy C, Liu Y, Needleman DS, Baranzoni GM, Feng P (2016) Advances in molecular serotyping and subtyping of Escherichia coli. Front Microbiol 7:1-8. https://doi.org/10.3389/ fmicb.2016.00644

Haines AN, Gauthier DT, Nebergall EE, Cole SD, Nguyen KM, Rhodes MW, Vogelbein WK (2013) First report of Streptococcus parauberis in wild finfish from North America. Vet Microbiol 166:270-275. https://doi.org/10.1016/j.vetmic.2013.05.002

Haines A, Nebergall E, Besong E, Council K, Lambert O (2016) Draft genome sequences for seven Streptococcus parauberis isolates from wild fish in the Chesapeake Bay 4:1-2 . https://doi.org/10.1128/ genomeA.00741-16.Copyright

Jiang S, Wang LEI, Reeves PR (2006) Molecular characterization of Streptococcus pneumoniae type $4,6 \mathrm{~B}, 8$ and 18C capsular polysaccharide gene clusters. Infect Immun 69:1244-1255. https://doi.org/ 10.1128/IAI.69.3.1244

Kanai K, Yamada M, Meng F, Takakahashi I, Nagano T (2009) Serological differentiation of Streptococcus parauberis strains isolated from cultured Japanese flounder in Japan. Fish Pathol 44:3339

Kanai K, Tu C, Katayama N, Suga K (2015) Existence of subserotypes in Streptococcus parauberis serotype I. Fish Pathology 50:75-90

Kannika K, Pisuttharachai D, Srisapoome P, Wongtavatchai J, Kondo H, Hirono I, Unajak S, Areechon N (2017) Molecular serotyping, virulence gene profiling and pathogenicity of Streptococcus agalactiae isolated from tilapia farms in Thailand by multiplex PCR. J Appl Microbiol 122:1497-1507. https://doi.org/10.1111/jam.13447

Khan IU, Hassan AA, Lämmler AAC, Wolter W, Zschöck M (2003) Identification and epidemiological characterization of Streptococcus uberis isolated from bovine mastitis. J Vet Sci 4: 213-223

Morais V, Dee V, Suárez N (2018) Purification of capsular polysaccharides of Streptococcus pneumoniae : traditional and new methods. Front Microbiol 6:145. https://doi.org/10.3389/fbioe.2018.00145

Nho SW, Hikima JI, Cha IS, Bin PS, Bin JH, del Castillo CS, Kondo H, Hirono I, Aoki T, Jung TS (2011) Complete genome sequence and immunoproteomic analyses of the bacterial fish pathogen Streptococcus parauberis. J Bacteriol 193:3356-3366. https://doi. org/10.1128/JB.00182-11

Nho SW, Hikima JI, Park SB, Jang HB, Cha IS, Yasuike M, Nakamura Y, Fujiwara A, Sano M, Kanai K, Kondo H, Hirono I, Takeyama H, Aoki T, Jung TS (2013) Comparative genomic characterization of three Streptococcus parauberis strains in fish pathogen, as assessed by wide-genome analyses. PLoS One 8:1-13. https://doi.org/10. 1371/journal.pone.0080395 
Oguro K, Yamane J, Yamamoto T, Ohnishi K, Oshima S-I, Imajoh M (2014) Draft genome sequence of Streptococcus parauberis strain SK-417, isolated from diseased Sebastes ventricosus in Kagoshima, Japan. Genome Announc 2:e0453-e00414. https://doi.org/10. 1128/genomeA.00453-14

Ohbayashi K, Oinaka D, Hoai TD, Yoshida T, Nishiki I (2017) PCRmediated identification of the newly emerging pathogen Lactococcus garvieae serotype II from Seriola quinqueradiata and S. dumerili. Fish Pathol 52:46-49. https://doi.org/10.3147/jsfp.52. 46

Okura M, Takamatsu D, Maruyama F, Nozawa T, Nakagawa I, Osaki M (2013) Genetic analysis of capsular polysaccharide synthesis gene clusters from all serotypes of Streptococcus suis : potential mechanisms for generation of capsular variation. Appl Environ Microbiol 79:2796-2806. https://doi.org/10.1128/AEM.03742-12

Park MA, Kwon MG, Hwang JY, Jung SH, Kim D-W, Park J-Y, Kim JS, Na Y-J, Kim M-Y, Kim D-S, Chae S-H, Seo JS (2013) Genome sequence of Streptococcus parauberis strain KCTC11980, isolated from diseased Paralichthys olivaceus. Genome Announc 1:5-6. https://doi.org/10.1128/genomeA.00780-13

Pazos F, Santos Y, Macías AR, Núñez S, Toranzo AE (1996) Evaluation of media for the successful culture of Flexibacter maritimus. J Fish Dis 19:193-197. https://doi.org/10.1111/j.1365-2761.1996. tb00701.x

Rochat T, Fujiwara-nagata E, Calvez S, Dalsgaard I, Madsen L, Calteau A, Lunazzi A, Nicolas P, Wiklund T, Bernardet J, Duchaud E, Lawrence ML (2017) Genomic characterization of Flavobacterium psychrophilum serotypes and development of a multiplex PCRbased serotyping scheme. Front Microbiol 8:1752. https://doi.org/ 10.3389/fmicb.2017.01752

Shoemaker CA, Xu D, García JC, Lafrentz BR (2017) Capsular typing of Streptococcus agalactiae (Lancefield group B streptococci) from fish using multiplex PCR and serotyping. Bull Eur Assoc Fish Pathol 37:2017

Thompson J, Higgins D, Gibson T (1994) CLUSTAL W: improving the sensitivity of progressive multiple sequence alignment through sequence weighting, position-specific gap penalties and weight matrix choice. Nucleic Acids Res 22:4673-4680

Toranzo AE, Cutrin JM, Nunez S, Romalde JL, Barja JL (1995) Antigenic characterization of Enterococcus strains pathogenic for turbot and their relationship with other Gram-positive bacteria. Dis Aquat Org 21:187-191. https://doi.org/10.3354/dao021187

Torres Corral Y, Fernández Álvarez C, Santos Y (2019) Proteomic and molecular fingerprinting for identification and tracking of fish pathogenic Streptococcus. Aquaculture 498:322-334. https://doi.org/10. 1016/j.aquaculture.2018.08.041

Torres-Corral Y, Fernández-Álvarez C, Santos Y (2019) High - throughput identification and quantification of Vagococcus salmoninarum by SYBR Green I - based real - time PCR combined with melting curve analysis. J Fish Dis 42:1359-1368. https://doi.org/10.1111/ jfd. 13053

Tu C, Suga K, Kanai K (2015a) Structure of genetic loci for capsular polysaccharide biosynthesis in Streptococcus parauberis isolated from japanese flounder. Fish Pathol 50:192-199

Tu C, Suga K, Kanai K (2015b) A multiplex PCR assay for differentiation of Streptococcus parauberis serotypes. Fish Pathol 50:213-215

Wang K, Fan W, Cai L, Huang B, Lu C (2011) Genetic analysis of the capsular polysaccharide synthesis locus in 15 Streptococcus suis serotypes. FEMS Microbiol Lett 324:117-124. https://doi.org/10. 1111/j.1574-6968.2011.02394.x

Wiklund T, Madsen L, Bruun MS, Dalsgaard I (2000) Detection of Flavobacterium psychrophilum from fish tissue and water samples by PCR amplification. J Appl Microbiol 88:299-307. https://doi. org/10.1046/j.1365-2672.2000.00959.x

Williams AM, Collins MD (1990) Molecular taxonomic studies on Streptococcus uberis types I and II. Description of Streptococcus parauberis sp nov. J Appl Bacteriol 68:485-490

Publisher's note Springer Nature remains neutral with regard to jurisdictional claims in published maps and institutional affiliations. 